\title{
Preface
}

\section{Epicardial Interventions in \\ Electrophysiology: \\ Transformation to an \\ Established Approach}

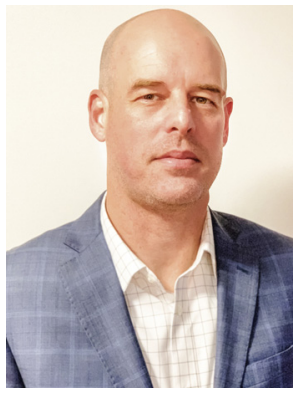

Jason S. Bradfield, MD

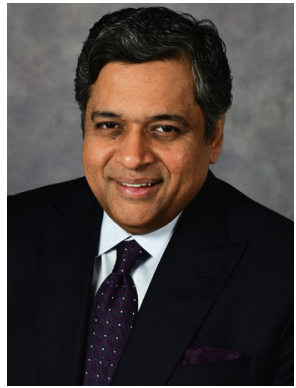

Kalyanam Shivkumar, MD, PhD

Editors
Percutaneous epicardial interventions in electrophysiology were initially utilized as a bail-out strategy to manage ventricular tachycardia in difficult-to-ablate nonischemic cardiomyopathy patients, in particular, patients with Chagas disease who had a predilection for epicardial and midmyocardial substrates. Subsequent clinical studies demonstrated the benefit of this approach in additional subsets of patients and led to more widespread use. This topic was reviewed by Cardiac Electrophysiology Clinics a decade ago.

The current literature in this area has grown, and it now supports the use of epicardial mapping/ablation in nonischemic cardiomyopathy, subsets of ischemic cardiomyopathy (failed endocardial ablation and/or transmural scar), and even in some patients with idiopathic ventricular tachycardia, supraventricular tachycardia, and atrial fibrillation. For this reason, Clinical Cardiac Electrophysiology fellowship trainees interested in joining or developing complex ablation programs have been drawn to learning epicardial interventions. In addition, in recent years with the development of percutaneous left atrial appendage interventions, practitioners have been further drawn to this skill set. Interest will undoubtedly continue to grow with future novel interventions for ablation, pacing, defibrillation, structural interventions, drug delivery, and gene therapy, among others.
In this series, we provide a much needed, stateof-the art review of the spectrum of epicardial interventions in electrophysiology and beyond. We are honored to have international leaders in the field contribute to the effort. Epicardial anatomy and access techniques are essential foundational reading. Subsequent comprehensive articles assess mapping and ablation of the spectrum of supraventricular and ventricular arrhythmias, novel mapping and ablation techniques, as well as the utility of perioperative imaging, optimization of ablation lesion technology, and the associated risks of these interventions. Finally, the future of epicardial interventions is discussed to complete the series. We thank the authors for crystalizing the state-of-the-art for the reader.

Jason S. Bradfield, MD

UCLA Cardiac Arrhythmia Center 100 medical Plaza, Suite 660 Los Angeles, CA 90095, USA

Kalyanam Shivkumar, MD, PhD UCLA Cardiac Arrhythmia Center 100 UCLA Medical Plaza, Suite 660 Los Angeles, CA 90095, USA

E-mail addresses: JBradfield@mednet.ucla.edu (J.S. Bradfield) kshivkumar@mednet.ucla.edu (K. Shivkumar) 